\title{
Review
}

Arndt Vogel* and Martha M. Kirstein

\section{First-line molecular therapies in the treatment of metastatic colorectal cancer - a literature-based review of phases II and III trials}

https://doi.org/10.1515/iss-2018-0012

Received March 23, 2018; accepted April 3, 2018; previously published online May 7, 2018

\begin{abstract}
Introduction: Metastatic colorectal cancer (mCRC) is one of the most common cancers and the second leading cause of cancer worldwide. With the improvement of systemic and operative therapies, median overall survival (mOS) reached 30 months or longer. Here, we will review the use of the anti-vascular endothelial growth factor (VEGF) and anti-epidermal growth factor receptor (EGFR) antibodies in combination with doublet and triplet chemotherapy in patients with borderline and primary unresectable mCRC. Methods: Phases II and III trials were included in investigating chemotherapy in the first-line in combination with an anti-VEGF(R) or anti-EGFR in a cohort of patients with mCRC.
\end{abstract}

Results: The VEGF-antibody bevacizumab has improved progression-free survival (PFS) in several phase III trials in combination with a chemotherapy doublet. More recently, a higher efficacy has been demonstrated in combination with an intensified chemotherapy including 5-fluoropyrimidine (5-FU), oxaliplatin, and irinotecan within the phase III TRIBE study. Similarly, high resectability rates have been shown in the phase II Olivia trial for patients with liver-limited disease with an intensified chemotherapeutic regime. However, this increase in efficacy was accompanied by an increase in toxicity as well. The efficacy of the EGFR-antibodies cetuximab and panitumumab has been shown in several phase III trials, but their use is restricted to patients whose tumors are RAS wildtype (WT). The phase II trials, CELIM and PLANET, demonstrated

*Corresponding author: Prof. Dr. med. Arndt Vogel, Department of Gastroenterology, Hepatology and Endocrinology, Hannover Medical School, Carl-Neuberg-Str. 1, 30625 Hannover, Germany, Phone: 00495325119590, E-mail: vogel.arndt@mh-hannover.de Martha M. Kirstein: Department of Gastroenterology, Hepatology and Endocrinology, Hannover Medical School, Hannover, Germany a favorable long-term survival for patients with initially non-resectable colorectal liver metastases who respond to conversion therapy with EGFR-antibodies and undergo secondary resection. The CLGB and FIRE-3 trials delivered an inconsistent finding whether anti-VEGF or -EGFR treatment is the better option in the first-line setting. However, there is increasing evidence from post hoc analyses of prospective clinical trials that patients with left-sided tumors benefit from EGFR-directed combination therapy in terms of prolongation of OS and PFS compared with limited, if any, benefit for those with right-sided tumors.

Conclusion: Both anti-VEGF- and anti-EGFR-directed therapies represent efficient treatment options for patients with mCRC in the first line. For patients with RAS WT, leftsided tumor anti-EGFR-based treatment is recommended. Intensified regimens can be offered initially to unresectable patients in order to achieve resectability at a price of higher toxicity.

Keywords: bevacizumab; cetuximab; colorectal cancer; panitumumab.

\section{Introduction}

Colorectal cancer (CRC) is one of the most common cancers and the second leading cause of cancer worldwide [1]. Approximately $25 \%$ of newly diagnosed patients have already developed metastases, and $50 \%$ of all CRC patients will develop metastases over time as the disease progresses [2]. Overall survival (OS) for patients with metastatic colorectal cancer (mCRC) has markedly improved within the last two decades, and the most recent generation of randomized clinical trials has yielded median OS (mOS) durations of 30 months or longer. There are numerous reasons including the increase in resection rates, emerging treatment options in a therapeutic sequence but also improvement of first-line therapies. The standard first-line therapy consists of a combination of chemotherapy with targeted agents. The chemotherapeutic regimens are based on fluoropyrimidine/leucovorin (5-FU/LV) or 
capecitabine with or without irinotecan, oxaliplatin, or in combination with both. First-line molecular therapies include monoclonal antibodies targeting the vascular endothelial growth factor (VEGF) - bevacizumab - and the epidermal growth factor receptor (EGFR) panitumumab and cetuximab. In contrast to bevacizumab, cetuximab and panitumumab are only efficient in a subset of patients whose tumors are wildtype (WT) for Kirsten rat sarcoma (KRAS) and Neuroblastoma rat sarcoma (NRAS WT) [3-5]. Next to molecular prediction, tumor localization is increasingly accepted for decision making for either anti-VEGF or anti-EGFR therapy. Novel therapeutic targets include programmed death protein 1 (PD-1) and BRAF based on the molecular profiles of the tumors. In this review, we will summarize the systemic treatment options in the first-line setting for patients with $\mathrm{mCRC}$. We will specifically focus the review on the use of the anti-VEGF and anti-EGFR antibodies in combination with doublet and triplet chemotherapy in patients with borderline and primary unresectable mCRC.

\section{Methods}

A literature research for phases II and III trials in mCRC was performed. A trial was eligible for this review if it comprised a cohort of mCRC patients who were treated with chemotherapy in the first-line in combination with an anti-VEGF(R) or anti-EGFR antibody either in the palliative setting or a potentially curative/downsizing setting. Phase I and retrospective data were excluded.

\section{Results}

\section{Anti-VEGF antibodies in first-line treatment}

\section{Anti-VEGF antibodies in combination with chemotherapy doublets}

The prolongation of progression-free survival (PFS) by the addition of bevacizumab to chemotherapy has been demonstrated within several phase III trials. Hurwitz et al. [6] analyzed bevacizumab vs. placebo in combination with irinotecan and 5-FU/LV (IFL) as first-line therapy. In this study, the primary endpoint OS as well as the secondary endpoints PFS and overall response rate (ORR) were significantly improved by bevacizumab [7]. The N016966 trial $(n=1401)$ compared oxaliplatin with capecitabine or 5-FU/LV (CAPOX or FOLFOX) plus bevacizumab or placebo
[8]. Bevacizumab significantly improved the primary endpoint PFS and non-significantly the secondary endpoint OS. Stathopoulos et al. investigated bevacizumab in combination with 5-FU/LV and irinotecan (FOLFIRI) in a small, single-center trial with only 222 patients. There was no statistical difference in the primary endpoint OS between the arms. However, treatment was stopped after eight cycles of therapy rather than until disease progression [9].

The MAX study $(n=471)$ analyzed the efficacy of bevacizumab in combination with capecitabine-based chemotherapy in first-line [10]. In this study, the addition of bevacizumab significantly improved the primary endpoint PFS. The AVEX study $(n=280)$ provided evidence for a safe and efficient administration of capecitabine with bevacizumab in elderly patients, where PFS and ORR were significantly improved [11]. OS was also improved, but the difference between the two arms was not statistically significant - mOS was 20.7 months in the combination arm compared with 16.8 months in the capecitabine arm. Very recently, the first data from the AIO KRK-0110 study $(n=421)$ have been reported, which compared the sequential application of 5-FU/LV-bevacizumab followed by the addition of irinotecan at first progression to the direct application of all three drugs in an elderly population of patients with untreated mCRC [12]. In this trial, the reduced initial treatment intensity failed to meet non-inferiority limits compared with the initial standard-of-care, full-intensity treatment. ORR also favored the initial irinotecan arm (36.8\% vs. 53.6\%). Moreover, among patients with RAS WT tumors, time to failure of strategy (TFS) was 8.6 months for the sequential arm and 11.8 months for the full-intensity treatment, meeting the non-inferiority, while there was no significant difference among patients with RAS-mutant tumors. Similarly, there was a significant 5-month advantage for the more intensive first-line treatment in RAS WT tumors (28.5 months vs. 23.5 months), whereas mOS was similar between both arms for patients with RAS-mutant tumors. Overall, these data suggest that sequential therapy cannot be recommended in patients with RAS/BRAF WT mCRC. However, sequential bevacizumab-based therapy could be discussed as an option in elderly patients with RAS mutant mCRC.

\section{Anti-VEGF antibodies in combination with chemotherapy triplets}

An intensified chemotherapy in terms of 5-FU/LV, oxaliplatin, and irinotecan (FOLFOXIRI) plus bevacizumab has been analyzed within the phase III TRIBE study ( $n=508)$ in patients $\leq 70$ years old at a good performance 
status [Eastern Co-operative Oncology Group (ECOG) performance status $\leq 2$ ] [13] (Table 1). In line with previous findings, treatment with FOLFOXIRI or FOLFOXIRI-bevacizumab was feasible in this multicenter study with no new safety signals. However, in all trials with triplet chemotherapy, grade 3/4 toxicity (neuropathy, diarrhea, neutropenia) is increased compared to the doublet chemotherapy. FOLFOXIRI-bevacizumab significantly improved OS compared to FOLFIRI-bevacizumab from 25.8 months to 29.8 months with a tolerable safety profile. The median PFS (mPFS) was prolonged by 2.4 months, reaching 12.1 months in the FOLFOXIRI group. The response rate was 53.1\% with FOLFIRI, compared with $65.1 \%$ with FOLFOXIRI-bevacizumab. The higher ORR, however, did not translate into a higher rate of R0 resection of metastases, which was not significantly different in both treatment groups (12\% vs. $15 \%$ in the experimental group). Subgroup analyses did not reveal any interaction between baseline characteristics and treatment effect, which was consistent for all subgroups including patients with WT and mutant RAS or BRAF tumors. The results of the TRIBE study have been recently supported by the phase II CHARTA trial $(\mathrm{n}=250)$. In this study, outcomes with FOLFOX-bevacizumab vs. FOLFOXIRI-bevacizumab was compared in 242 patients. FOLFOXIRI-bevacizumab was administered at the same dose and schedule as in the TRIBE study [14]. FOLFOXIRI-bevacizumab significantly improved PFS at 9 months: $68 \%$ vs. $56 \%$ with FOLFOXbevacizumab with an mPFS of 12 months and 10 months, respectively, representing a $20 \%$ reduction in risk with FOLFOXIRI-bevacizumab. Response rates were $70 \%$ for FOLFOXIRI and $60 \%$ for FOLFOX. The overall survival data are not yet mature. At the time of the first analysis in 2017, mOS was 28 months with FOLFOXIRI-bevacizumab and
24 months with FOLFOX-bevacizumab, with the curves separating early in favor of the FOLFOXIRI-bevacizumab regimen and with partial crossover occurring beyond 2 years [14]. Finally, the triplet chemotherapy in combination with bevacizumab was analyzed in patients with initially non-resectable liver metastases from colorectal cancer [15]. In the randomized phase II OLIVIA trial $(n=80)$, the efficacy of bevacizumab plus modified FOLFOX6 was compared to FOLFOXIRI-bevacizumab similar to the CHARTA study. Resectability was evaluated by multidisciplinary review, and non-resectability was defined as $\geq 1$ of the following criteria: no possibility of upfront R0/R1 resection of all lesions; $<30 \%$ residual liver volume after resection; and metastases in contact with major vessels of the remnant liver. The ORRs were $81 \%$ with FOLFOXIRI-bevacizumab and $62 \%$ with mFOLFOX6-bevacizumab, whereas mPFS was 18.6 months and 11.5 months, respectively. In agreement with the higher response rate, the overall resection rate was $61 \%$ with FOLFOXIRI-bevacizumab and $49 \%$ with mFOLFOX6-bevacizumab.

All three studies together indicate that the addition of a third chemotherapeutic agent to a chemotherapy doublet offered improved efficacy. The higher ORR achieved in OLIVIA support the hypothesis that patients with liver-limited disease respond better. The multi-center, phase II MOMA study $(\mathrm{n}=232)$ currently explores the role of maintenance with bevacizumab alone compared with bevacizumab plus metronomic chemotherapy following a 4-month induction therapy with FOLFOXIRI-bevacizumab [16]. In this study, patients were randomized to induction therapy with FOLFOXIRI-bevacizumab for 4 months, followed by bevacizumab until disease progression or by bevacizumab

Table 1: Randomized controlled trials investigating triple chemotherapies in combination with targeted therapies as first-line therapies in patients with metastatic colorectal cancer.

\begin{tabular}{|c|c|c|c|c|c|}
\hline Study & $\begin{array}{l}\text { Patients } \\
\text { (ITT population) }\end{array}$ & Study name & $\begin{array}{l}\text { Overall response } \\
\text { rate }(\%)\end{array}$ & $\begin{array}{l}\text { mPFS } \\
\text { (months) }\end{array}$ & $\begin{array}{l}\text { mOS } \\
\text { (months) }\end{array}$ \\
\hline CHARTA & $\mathrm{n}=250$ (total); & Arm A: FOLFOX + BEVACIZUMAB & Arm A: 60 & Arm A: 9.8 & - \\
\hline Phase II & $1: 1$ randomization & Arm B: FOLFOXIRI + BEVACIZUMAB & Arm B: 70 & Arm B:12 & \\
\hline TRIBE & $n=252 / 256$ & Arm A: FOLFOXIRI + BEVACIZUMAB & Arm A: 65 & Arm A: 12.3 & Arm A: 29.8 \\
\hline Phase III & $1: 1$ randomization & Arm B: FOLFIRI + BEVACIZUMAB & Arm B: 54 & Arm B: 9.7 & Arm B: 25.8 \\
\hline MOMA & $\mathrm{n}=117 / 115$ & Arm A: BEVACIZUMAB & Arm A: 68 & Arm A: 9.5 & - \\
\hline Phase II & 1:1 randomization & $\begin{array}{l}\text { Arm B: BEVACIZUMAB + metroCT } \\
\text { Maintanace after induction with } \\
\text { FOLFOXIRI + BEVACIZUMAB }\end{array}$ & $\begin{array}{l}\text { Arm B: } 58 \\
\text { After induction } \\
\text { therapy }\end{array}$ & Arm B: 10.6 & \\
\hline STEAM & $\mathrm{n}=280$ (total); & Arm A: FOLFOXIRI + BEVACIZUMAB & Arm A: 72 & Arm A: 11.7 & - \\
\hline Phase II & 1:1:1 randomization & $\begin{array}{l}\text { Arm B: sFOLFOXIRI+ BEVACIZUMAB } \\
\text { Arm C: FOLFOX + BEVACIZUMAB }\end{array}$ & $\begin{array}{l}\text { Arm B: } 73 \\
\text { Arm C: } 62\end{array}$ & $\begin{array}{l}\text { Arm B: } 10.7 \\
\text { Arm C: } 9.3\end{array}$ & \\
\hline
\end{tabular}

metroCT, Capecitabine $500 \mathrm{mg} /$ tid and cyclophosphamide $50 \mathrm{mg} /$ day per os; sFOLFOXIRI, alternating FOLFOX and FOLFIRI every 4 weeks. 
in combination with metronomic capecitabine and oral cyclophosphamide until progressive disease (PD). ORR with FOLFOXIRI-bevacizumab was $63 \%$, and in the liver-limited subgroup, the secondary resection rate was $49 \%$. The addition of metronomic chemotherapy to the maintenance with bevacizumab, however, did not significantly improve PFS, which was the primary endpoint indicating that 5-FU/LV-bevacizumab remains the preferred maintenance therapy. The impact of a chemotherapeutic triplet or doublet and the consequent re-induction options are currently being investigated within the phase III TRIBE-2 trial. This study investigates the first-line FOLFOX-bevacizumab followed by FOLFIRI-bevacizumab after disease progression or FOLFOXIRI-bevacizumab followed by the re-introduction of the same regimen after disease progression. The primary endpoint is to compare the efficacy of the two proposed treatment strategies in terms of the PFS following reinduction [17].

The concurrent use of triplet chemotherapy is mainly feasible in a fit patient in a good performance status. The randomized, open-label, phase II STEAM (=93) trial evaluated concurrent FOLFOXIRI and sequential FOLFOXIRI (FOLFOX and FOLFIRI alternated every two cycles) as chemotherapy backbones in comparison to FOLFOX as standard comparator [18]. Patients in all arms received bevacizumab, which had to be continued beyond the first disease progression. The primary endpoint of this trial was ORR. The initial results from this exploratory study revealed an improved ORR and PFS with the FOLFOXIRI backbones. Among the 280 patients included in the trial, the ORRs reached $62 \%$ with FOLFOX-bevacizumab, $72 \%$ with the concurrent FOLFOXIRI-bevacizumab and, also $73 \%$ with the sequential FOLFOXIRI-bevacizumab. The not yet mature mPFS data revealed slightly improved preliminary durations of 11.7 months with concurrent FOLFOXIRI-bevacizumab and 10.7 months with sequential FOLFOXIRI-bevacizumab compared with 9.3 months with FOLFOX-bevacizumab. In agreement with the higher ORR, secondary liver resection rates were higher with concurrent FOLFOXIRI-bevacizumab compared with FOLFOX-bevacizumab (15.1\% vs. $7.4 \%)$. These rates were, however, only slightly higher with sequential FOLFOXIRI-bevacizumab compared to FOLFOX-bevacizumab (9.8\% vs. $7.4 \%)$. Overall, the modest increase in efficacy with the sequential FOLFOXFIRI-bevacizumab regimen came at the cost of slightly higher toxicity, but might be an interesting option for patients with borderline non-resectable or symptomatic disease, which are not regarded as candidates for the concurrent use of the triplet chemotherapy.

\section{Anti-EGFR antibodies in first-line treatment}

\section{Anti-EGFR antibodies in combination with chemotherapy doublets}

The efficacy of anti-EGFR treatment is restricted to patients whose tumors are RAS WT. In the first trials, antiEGFR therapy had been studied in patients with EGFRexpressing tumors, which has no predictive function as meanwhile shown. Similar to bevacizumab, several phase III trials for the EGFR-antibodies - cetuximab and panitumumab - have been published. However, the survival data are partially incoherent most likely due to the numerous post hoc analyses based on, over time emerging, refinement of molecular selection.

Overall, 179 patients with KRAS and 87 patients with RAS WT were evaluated in the OPUS trial $(n=337)$. In the extended analysis of RAS WT tumors, ORR was significantly improved by the addition of cetuximab to FOLFOX4. There was also a trend favoring the cetuximab arm in terms of PFS and OS in the RAS WT group. There was no evidence that patients with other RAS mutations benefited from cetuximab, and in the combined population of patients with any RAS mutation, a clear detrimental effect was associated with the addition of cetuximab to FOLFOX4 [19]. As first-line combination of EGFR-targeted therapies with FOLFIRI, the phase III CRYSTAL study $(n=1118)$ analyzed the efficacy of cetuximab in patients with EGFR-expressing tumors [20]. In the retrospectively ascertained KRAS WT population, the addition of cetuximab significantly improved OS, PFS, and ORR in patients with KRAS WT tumors [21]. OS increased in the RAS WT populations to 28.4 months. Moreover, a retrospective analysis of the study confirmed that adding cetuximab to the first-line FOLFIRI improved clinical outcomes and R0 resection rates in KRAS WT and RAS WT MCRC patients with LLD as well as in those with non-LLD [22]. In a pooled analysis on the combined population of patients evaluable for KRAS mutation status from the Opus and Crystal studies, OS, PFS, and ORR were all significantly improved by cetuximab [23]

The three-arm MRC COIN study $(\mathrm{n}=1630)$ evaluated cetuximab in combination with FOLFOX/CAPOX continuously or intermittently in patients with KRAS WT tumors [4]. Cetuximab increased ORR, but did not improve PFS or OS in KRAS WT patients. The OS was still similar in the retrospective analysis of the COIN study, including 581 patients with RAS WT tumors. Another first-line combination of oxaliplatin-based chemotherapy with cetuximab was investigated within the three-arm NORDIC-VII study $(n=566)$ [24]. Arm A received FLOX, whereas arm 
$\mathrm{B}$ received FLOX-cetuximab continuously. In arm C, FLOX was usually stopped after 16 weeks of treatment, and cetuximab was continued as maintenance therapy. Endpoints were not significantly changed in the intentionto-treat (ITT) population and irrespective of the KRAS mutational status. In patients with KRAS WT tumors, cetuximab did not provide any additional benefit compared with FLOX alone, and OS was similar in all three arms.

The phase III PRIME study ( $\mathrm{n}=1183)$ evaluated panitumumab in combination with FOLFOX as first-line treatment $[5,25,26]$. In contrast to cetuximab, panitumumab in combination with FOLFOX significantly improved not only the primary endpoint PFS but also the secondary endpoint OS in patients with KRAS WT tumors. In patients with RAS WT, the benefit in OS provided by panitumumab increased to 6 months to an mOS of 26 months in patients with RAS WT tumors. In the RAS mutant groups receiving panitumumab, OS was significantly shorter compared to the placebo [5].

Several studies evaluating the efficacy of cetuximab in combination with chemotherapy in patients with liver-limited disease were published (Table 2). The CELIM study ( $\mathrm{n}=114$ ) was one of the first studies, which analyzed the effectiveness of cetuximab with either FOLFOX6 or FOLFIRI in patients with non-resectable colorectal liver metastases [27]. The primary endpoint was tumor response assessed by the Response Evaluation Criteria In Solid Tumours (RECIST). A retrospective, blinded surgical review of patients with radiological images at both baseline and during treatment was done to assess objectively any changes in resectability. Non-resectability was defined as having five or more liver metastases or metastases that were viewed as technically non-resectable by the local liver surgeon and radiologist on the basis of inadequate future liver remnant, or one of the following criteria: infiltration of all hepatic liver veins; infiltration of both hepatic arteries or both portal vein branches. The ORR was noted in $68 \%$ in patients treated with FOLFOX, and $57 \%$ treated with FOLFIRI R0 resection was possible in $38 \%$ and $30 \%$ of the patients in each group, respectively. In a retrospective analysis of response by KRAS status, the response was $70 \%$ in patients with KRAS WT tumors compared to $41 \%$ in patients with KRAS-mutated tumors. In patients with interventions, the median time to resection or exploration was 5.1 months, and the median number of treatment cycles before intervention was eight. Interestingly, paired baseline and follow-up scans were available for $64 \%$ of patients, which were evaluated by seven surgeons. The voting pattern of surgeons showed considerable inter-individual variation in the decision-making

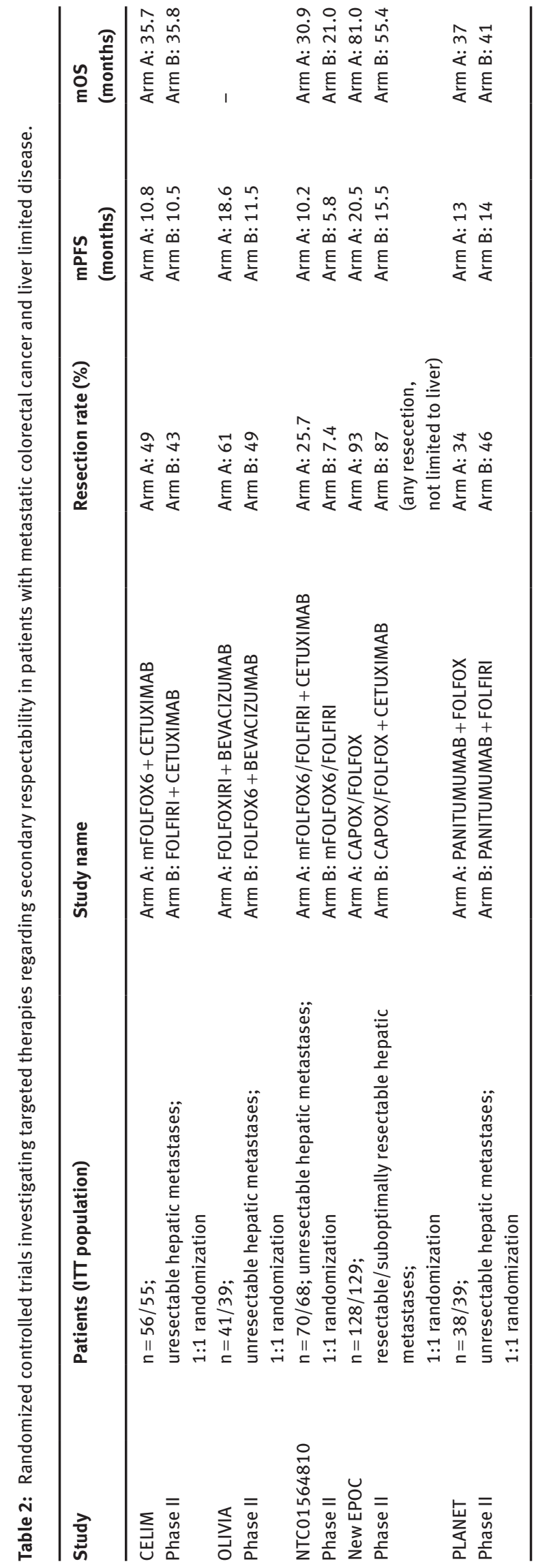


process, but overall, $60 \%$ of the patients were judged to be resectable after systemic therapy, compared with $32 \%$ of the patients at baseline. The mPFS in this study war is very similar between both arms with 10.8 and 10.5 months [28]. Moreover, recurrent disease was evident in all patients included in the study. The mOS was also very similar in both arms with 35.7 months and 29 months, respectively. As expected, patients who underwent R0 resection achieved a better mOS with 53.9 months compared to those who did not (21.9 months). The median disease-free survival for R0 resected patients was 9.9 months, and the 5 -year OS rate was $46.2 \%$. In a similar setting, the efficacy of panitumumab in combination with either FOLFOX6 or FOLFIRI was investigated in patients with KRAS WT mCRC with multiple or non-resectable liver metastases in the PLANET study $(n=77)$ [29]. The primary endpoint in this study was ORR, while liver metastases resection rate, PFS, and OS were secondary endpoints. The ORR was 74\% in the FOLFOX 4 and 67\% in the FOLFIRI arm (RAS WT: $78 \% / 73 \%$ ). Of the patients, $45 \%$ and 59\% underwent surgical resection, respectively (RAS WT: 37\%/69\%), and the R0-R1 resection rates were 34\%/46\% (RAS WT: 26\%/54\%). Similar to the CELIM trial, the mPFS was $13 / 14$ months, and the mOS was 37/41 months. Both endpoints were longer in patients who showed an early tumor shrinkage (ETS) $\geq 30 \% / \geq 20 \%$ at week 8 and in patients that underwent surgery. Together, CELIM and PLANET confirm that the combination of EGFR antibodies with either FOLFOX or FOLFIRI is feasible in patients with liver-limited mCRC and that there are no significant differences in efficacy between the two regimens. Moreover, these studies demonstrate a favorable long-term survival for patients with initially non-resectable colorectal liver metastases who respond to conversion therapy and undergo secondary resection.

Subsequently, two studies more specifically addressed the question whether the addition of cetuximab to chemotherapy improves long-term outcome in patients with liver-limited disease. In the New EPOC study ( $n=257)$, the efficacy of perioperative oxaliplatin-based chemotherapy with or without cetuximab was evaluated in patients with potentially resectable liver metastases [30]. Previously, the EORTC-40983 study has shown that a perioperative chemotherapy with FOLFOX in patients with liver limited disease and good prognostic factors (one to four resectable liver metastasis, 52\% with only one metastases, $26 \%$ with two metastasis, $65 \%$ with metachrone metastasis) does not significantly improve the 5-year survival rate [31]. In the New EPOC study, patients who had received oxaliplatin as adjuvant therapy were permitted to be treated with irinotecan. Altogether, 51 patients received irinotecan-based and 182 patients oxaliplatin-based therapy. Surprisingly, the addition of cetuximab reduced the primary endpoint PFS, and there was also a significant shorter mOS despite a moderate improvement of response preoperatively [32]. The second study evaluated the efficacy of cetuximab in Chinese patients with synchronous non-resectable liver metastasis [33]. After resection of the primary tumors, patients with KRAS WT were randomly assigned to receive chemotherapy FOLFIRI or FOLFOX with or without cetuximab. The primary endpoint was the rate of patients converted to resection for liver metastases, which was significantly improved by the addition of cetuximab. In contrast to the New EPOC study, cetuximab also significantly improved OR, PFS, and OS regardless of the applied chemotherapy regimen. Together, these studies confirm the feasibility of EGFR-based therapy in patients with liver-limited disease, but do not provide unequal evidence that the addition of cetuximab improves long-term outcome.

\section{Anti-EGFR antibodies in combination with chemotherapy triplets}

Several trials explored the combination of EGFR antibodies in combination with triplet chemotherapy. In one of the first trials, a dose-escalation phase I study was performed with cetuximab in combination with FOLFOXIRI in patients with previously untreated $\mathrm{mCRC}$ and a WHO performance status of $0-1$ [34]. At dose level 3 (irinotecan $165 \mathrm{mg} / \mathrm{m}^{2}$ ), three patients experienced a DLT (diarrhea grade 3 and two patients with neutropenia grade 4), and the recommended dose for a phase II trial was determined as $125 \mathrm{mg} / \mathrm{m}^{2}$ irinotecan in combination with oxaliplatin, 5-FU/LV, and cetuximab. The most common grade $\geq 3$ toxicities were neutropenia (40\%), diarrhea (25\%), and acne-like rash (15\%). The dose-limiting toxicity was also observed in subsequent trials for the combination of FOLFOXIRI and EGFR antibodies [35, 36]. The triplet combination, however, showed promising clinical activity in several small phase II trials, specifically in terms of response rate and R0 secondary liver metastases resection in patients with initially non-resectable disease confined to the liver. Overall, the response rate of $71 \%$ to $89 \%$ was consistently higher compared to the combination with a doublet chemotherapy $[37,38]$. The mOS varied between 9.5 and 16 months and the mOS between 24.7 and 37 months in these studies. More recently, the first results of the VOLFI trial $(n=93)$ have been reported, which has specially evaluated the activity and safety of mFOLFOXIRI-panitumumab vs. FOLFOXIRI in ECOG 0-1, 
primarily non-resectable mCRC patients [39]. In this study, two cohorts were analyzed, cohort 1 with non-resectable $\mathrm{mCRC}$ and cohort 2 with patients, which were considered to have a chance of secondary resection of metastatic lesions. The primary endpoint of the study was ORR, and the secondary endpoints included a secondary resection rate in cohort 2, disease control rate, PFS, OS, toxicity, and quality of life. In agreement with the previous studies, ORR was significantly higher in the panitumumab arm with $85.7 \%$ compared to $54.5 \%$ in the control arm. The ORRs, however, were differential according to tumor location and higher in left-sided tumors compared to rightsided tumors. Secondary resections in cohort 2 were $60 \%$ in the panitumumab arm and $36.4 \%$ in the control arm. The PFS, OS, and quality of life data were still immature at the time of this initial report. Serious adverse advents grades $3-5$ occurred in $45.3 \%$ of the patients in the combination arm, but appeared manageable in the younger, fit patients with ECOG 0-1 in agreement with the previous single-arm studies. Overall, these data indicate that the addition of the EGFR antibodies to a triplet chemotherapy results consistently in high response rates and secondary resection of metastasis and may be a valid option for fit patients with limited, upfront non-resectable metastasis. However, there is, so far, no clear evidence that the addition of EGFR antibodies to mFOLFOXIRI also improves other important endpoints such as PFS and OS in patients with potentially never resectable disease justifying the increased toxicity of this intensive regimen.

\section{Anti-VEGF vs. anti-EGFR: head to head comparison}

There are two phase III and one phase II head-to-head trials comparing anti-VEGF with anti-EGFR therapy in the first-line setting: FIRE-3, CALGB/SWOG 80405, and PEAK, respectively.

The phase III FIRE-3 study $(\mathrm{n}=592)$ compared the efficacy in terms of objective response of FOLFIRI-cetuximab vs. FOLFIRI-bevacizumab in patients with KRAS WT tumors as initial treatment. The study failed to show a significant difference of ORR and also of PFS. However, the secondary endpoint OS was significantly prolonged with 28.7 months for the cetuximab arm compared to 25 months for the bevacizumab arm [40]. Similarly, in a post hoc analysis of the final RAS WT population, the mOS was significantly better in the FOLFIRI-cetuximab group with 33.1 months compared to 25.0 months in the FOLFIRI-bevacizumab group, although the investigatorassessed ORR and PFS remained similar between both treatment groups [41]. A centralized radiological review of CT scans was done in a post hoc analysis to assess the objective response according to RECIST 1.1, early tumor shrinkage (ETS), depth of response, duration of response, and time to response in the final RAS WT subgroup. This radiological review allowed to correlate the OS benefit of FOLFIRI-cetuximab in the extended RAS WT with superior response-related outcome parameters, such as ETS and depth of response. In an additional post hoc analysis, the number of patients who would have been candidates for surgery up front were compared with the number of patients who were considered "resectable" at best response following systemic treatment by a panel of eight experienced surgeons and three medical oncologists [42]. Overall, surgical intervention was retrospectively considered possible in $22 \%$ of the patients at baseline and in $53 \%$ at best response after treatment with FOLFIRI in combination with either cetuximab or bevacizumab, with no significant difference between both arms. Interestingly, the recommendations for surgery (yes vs. no) showed moderate consistency (for/against intervention) at baseline and substantial higher consistency at best response. An actual secondary resection out of these potentially resectable patients occurred in only $16 \%$ with a higher resection rate in academic hospitals. The mOS was 51.3 months in patients who received surgery, 30.8 months in patients with resectable disease, who did not receive surgery, and 18.6 months in patients with never resectable disease. Overall, these data highlight the need for a continuous evaluation for metastatic resection in patients with upfront non-resectable disease.

The CALGB/SWOG 80405 trial $(\mathrm{n}=1137)$ is the largest head-to-head trial with 2334 patients randomized. Patients were treated with FOLFOX or FOLFIRI (by the investigator's discretion) in combination with cetuximab or bevacizumab [43]. The initial design included unselected mCRC patients but was amended after 1420 patients were accrued, to include only patients with KRAS WT tumors. Among the patients with KRAS WT tumors, $73 \%$ were treated with FOLFOX and the other $27 \%$ with FOLFIRI. In contrast to the FIRE-3 study, there was no difference in OS with 29.0 months for the bevacizumab arm and 29.9 months for the cetuximab arm $(H R=0.92)$. In the expanded RAS WT population, the median OS were 31.2 and 32 months for the bevacizumab and the cetuximab arms, respectively [44]. Likewise, PFS did not differ between treatments (10.8 months for bevacizumab and 10.4 months for cetuximab). The analysis according to the chemotherapy backbone also did not reveal any significant differences. The subgroup analysis of the patients treated with FOLFIRI did not confirm the results of the 
AIO FIRE-3 study, and neither PFS nor OS was different between the cetuximab and the bevacizumab arms when combined with FOLFIRI. A significant higher ORR was achieved in the cetuximab arm in the extended RAS population (68.6\% vs. 53.6\%). A resection was overall possible in 180 KRAS exon 2 WT patients and a R0 resection in 82 patients (30\%) in the cetuximab arm and in 50 patients (15\%) in the bevacizumab arm. The mOS of these patients was 64.7 months, which was not significantly different between arms.

The phase II PEAK trial $(\mathrm{n}=285)$ compared FOLFOXpanitumumab or -bevacizumab in the first-line treatment of mCRC patients with KRAS WT tumors with a primary endpoint of PFS. The ITT analysis did not show significant differences in terms of PFS, OS, or ORR in the KRAS WT population. In the RAS WT population, an improvement of mPFS (13.0 vs. 9.5 months) and of mOS (41.3 vs. 28.9 months) in favor of the panitumumab-treated patients was observed [45]. Similar to the FIRE-3 study, more patients treated with panitumumab achieved an ETS at week 8 compared with bevacizumab and tumor shrinkage of $30 \%$ or more was associated with longer mPFS and mOS compared to when patients had tumor shrinkage below 30\%.

Taken together, there is still no clear evidence for choosing an anti-angiogenic over an anti-EGFR treatment or vice versa for a combination with the commonly used chemotherapy regimens in the first-line setting in patients with mCRC when considering PFS and OS as endpoint. The results suggest that the benefit is higher in terms of ORR in patients treated with EGFR antibodies compared to bevacizumab in the RAS WT population.

\section{Primary tumor localization and efficacy of anti-EGFR and anti-VEGF-directed therapy}

There is increasing evidence that mCRC is a heterogeneous disease and that tumors arising from different sides of the colon (left vs. right) have different distinct clinical and molecular characteristics. Approximately two-thirds of CRCs are derived from the left side and the remaining one-third from the right side. Right-sided tumors are more common in women and are likely to be diploid, more commonly associated with poor prognostic indicators such as RAS and BRAF tumor mutations, microsatellite instability (MSI), CpG island methylator phenotype (CIMP)-high, and mucinous histology [46]. The left-sided tumors are more common in men, more commonly associated with chromosomal instability, KRAS, p53, HER1 and HER2 gene amplification, aneuploidy, and gene expression profiles consistent with sensitivity to EGFR-targeted antibody therapy [47, 48] A pooled analysis of six trials (FIRE3, CALGB 80405, PEAK, CRYSTAL, PRIME, and 20050181) has been performed to investigate the prognostic and predictive influence of the localization of the primary tumor in patients with non-resectable RAS WT mCRC [49]. The primary tumor location and RAS status were available for 2159 of the 5760 patients with 515 right-sided and 1644 leftsided tumors. The analysis revealed a significant worse prognosis for patients with right-sided tumors compared with those with left-sided tumors in terms of mOS, mPFS, and ORR confirming the evidence from a recent metaanalysis with 66 trials [50]. In terms of predictive value, a significant benefit for EGFR-directed combination therapy was observed in patients with left-sided tumors for OS and PFS, compared with limited, if any, benefit for those with right-sided tumors. For the ORR, there was a trend for a greater benefit for chemotherapy plus EGFR antibody therapy in the patients with left-sided tumors compared with those with right-sided tumors. Specifically, in the FIRE-3 and CALGB 80405 studies, patients with left-sided tumors receiving cetuximab did significantly better than those receiving bevacizumab in combination with chemotherapy, while patients with right-sided RAS WT tumors benefit more from chemotherapy plus bevacizumab compared with cetuximab.

\section{Treatment decision in first-line therapy according to current guidelines}

The most recent European Society for Medical Oncology (ESMO) consensus guidelines define tumor characteristics, patient characteristics, and treatment characteristics as the drivers of decision making in the first-line treatment setting, as well as therapeutic goal differentiating between "disease stabilization" and the necessity for tumor volume reduction [51]. More recently, the authors argued that a distinction needs to be made between the treatment approaches for patients with right- vs. left-sided tumors [49]. Despite the retrospective data they recommend that for the majority of patients with left-sided RAS WT tumors, the preferred treatment option would be a chemotherapy doublet plus EGFR antibody therapy, independent of the treatment goal. For patients with right-sided RAS WT tumors, the preferred treatment option would depend on the treatment goal. For patients requiring cytoreduction, they recommend either a chemotherapy triplet such as FOLFOXIRI-bevacizumab or a doublet plus EGFR antibody therapy. For patients where disease stabilization is the goal, a chemotherapy doublet with or without bevacizumab would be the treatment of choice. 
BRAF mutations are a significant negative prognostic marker for patients with mCRC [52-57]. There is also accumulating evidence that BRAF mutations as a predictive biomarker for a lack of benefit for EGFR antibody therapy and two meta-analyses confirmed that the efficacy of EGFR antibody therapies is greater in patients with RAS WT/BRAF WT tumors compared to those with RAS WT/ BRAF-mutant tumors [58, 59]. The preferred treatment option for fit patients with BRAF-mutated tumors is FOLFOXIRI plus bevacizumab.

The recently published German S3 guideline also provides treatment recommendations based on tumor characteristics, patient characteristics, and treatment characteristics as the drivers of decision making in the first-line treatment setting [60]. In the palliative setting, the authors recommend, similar to ESMO, a chemotherapy doublet plus EGFR antibody therapy for patients with left-sided RAS WT tumors and a chemotherapy triplet for patients with BRAF-mutated tumors, which is generally only recommended for patients with a good performance status. Patients with a right-sided tumor should receive a chemotherapy doublet or triplet \pm bevacizumab. For patients with technically resectable metastasis, the preferred treatment is surgery, and a preoperative chemotherapy should only be considered in patients with synchronous metastasis or patients with an early relapse. If disease stabilization can be achieved in these patients, surgery should be performed as early as possible (after 2-3 months). An adjuvant/additive chemotherapy after resection of metastasis is not recommended.

The latest version of NCCN's Clinical Practice Guidelines in Oncology, Colon Cancer recommends, as the initial therapy for metastatic disease in patients appropriate for intensive therapy, a choice of five chemotherapy regimens: FOLFOX, FOLFIRI, CapeOx, infusional 5-FU/LV or capecitabine, or FOLFOXIRI with or without targeted agents [61]. They do not provide a clear preference between anti-EGFR therapy or bevacizumab for left-sided tumors. Similar to ESMO, the panel concluded that cetuximab and panitumumab confer little, if any, benefit to patients with metastatic CRC if the primary tumor originated on the right side, and they do not recommend anti-EGFR therapy for right-sided tumors in the first-line setting.

\section{Perspective}

Several biomarker-driven studies are currently being performed, which will tailor the treatment more to the molecular profile of the patients in the future. The FOCUS-4 is such a molecularly driven randomized trial for patients with mCRC (EudraCT 2012-005111-12). A biomarker panel analysis [BRAF, KRAS, NRAS, phosphatase and tensin homolog (PTEN), phosphoinositide-3-kinase (PI3KCA), and mismatch repair (MMR)] will be performed during the first 16 weeks of induction therapy. Patients with controlled disease will be randomized according to five molecular subtypes: BRAF mutant, PI3KCA subtype (mutation of PI3KCA gene or loss of PTEN protein), RAS mutant, RAS WT, and non-classified subtype. Each molecularly stratified trial aims to compare a novel intervention to placebo or standard care.

The MODUL trial (NCT02291289) is a biomarkerdriven maintenance treatment randomized study. All patients will receive 4 months of induction therapy with FOLFOX-bevacizumab. Subsequently, patients with controlled disease will be separated into cohorts for maintenance therapy according to their mutational status. The control arm in all cohorts will be fluoropyrimidine with bevacizumab. In cohort one, BRAF-mutant patients will be randomized to receive fluoropyrimidine with cetuximab and vemurafenib. In cohort two, BRAF WT patients will be randomized to receive 5-FU/LV or capecitabine, bevacizumab, and atezolizumab; in cohort three, participants with Her2/neu-positive tumors will be randomized to receive capecitabine, trastuzumab, and pertuzumab; in cohort four (no biomarker group), patients will be randomized to receive cobimetinib and atezolizumab.

\section{Conclusion}

Both anti-VEGF- and anti-EGFR-directed therapies represent efficient treatment options for patients with mCRC in the first line. For patients with unresectable RAS WT and left-sided tumors, anti-EGFR-based treatment is recommended. Patients with right-sided and/or BRAFmutated tumors should preferentially be treated with bevacizumab in combination with chemotherapy doublets or triplets. Resection rates of liver metastasis for patients with initially unresectable disease are low, but operability should be critically re-evaluated following initiation of chemotherapy. Surgery is the preferred treatment for patients with resectable metastasis. A neoadjuvant or adjuvant chemotherapy without targeted therapies could be discussed for patients with synchronous metastasis or patients with an early relapse. Patients with a need of regression due to symptoms, or with borderline resectable disease, should be offered intensified regimens such as FOLFOXIRI and bevacizumab or FOLFIRI plus anti-EGFR for left-sided tumors. 


\section{Author Statement}

Research funding: Authors state no funding involved. Conflict of interest: Authors state no conflict of interest. Informed consent: Informed consent is not applicable. Ethical approval: The conducted research is not related to either human or animal use.

\section{Author Contributions}

Arndt Vogel: conceptualization; data curation; writing original draft. Martha M. Kirstein: data curation; writing original draft.

\section{References}

[1] Siegel R, Ward E, Brawley 0, Jemal A. Cancer statistics, 2011: the impact of eliminating socioeconomic and racial disparities on premature cancer deaths. CA Cancer J Clin 2011;61:212-36.

[2] Lim HJ, Gill S, Speers C, Melosky B, Barnett J, Fitzgerald C, et al. Impact of irinotecan and oxaliplatin on overall survival in patients with metastatic colorectal cancer: a population-based study. J Oncol Pract 2009;5:153-8.

[3] De Roock W, Claes B, Bernasconi D, De Schutter J, Biesmans B, Fountzilas G, et al. Effects of KRAS, BRAF, NRAS, and PIK3CA mutations on the efficacy of cetuximab plus chemotherapy in chemotherapy-refractory metastatic colorectal cancer: a retrospective consortium analysis. Lancet Oncol 2010;11:753-62.

[4] Maughan TS, Adams RA, Smith CG, Meade AM, Seymour MT, Wilson RH, et al. Addition of cetuximab to oxaliplatin-based first-line combination chemotherapy for treatment of advanced colorectal cancer: results of the randomised phase 3 MRC COIN trial. Lancet 2011;377:2103-14.

[5] Douillard JY, Oliner KS, Siena S, Tabernero J, Burkes R, Barugel $M$, et al. Panitumumab-FOLFOX4 treatment and RAS mutations in colorectal cancer. N Engl J Med 2013;369:1023-34.

[6] Hurwitz H, Fehrenbacher L, Novotny W, Cartwright T, Hainsworth J, Heim W, et al. Bevacizumab plus irinotecan, fluorouracil, and leucovorin for metastatic colorectal cancer. N Engl J Med 2004;350:2335-42.

[7] Hurwitz HI, Fehrenbacher L, Hainsworth JD, Heim W, Berlin J, Holmgren $\mathrm{E}$, et al. Bevacizumab in combination with fluorouracil and leucovorin: an active regimen for first-line metastatic colorectal cancer. J Clin Oncol 2005;23:3502-8.

[8] Saltz LB, Clarke S, Diaz-Rubio E, Scheithauer W, Figer A, Wong R, et al. Bevacizumab in combination with oxaliplatinbased chemotherapy as first-line therapy in metastatic colorectal cancer: a randomized phase III study. J Clin Oncol 2008;26:2013-9.

[9] Stathopoulos GP, Batziou C, Trafalis D, Koutantos J, Batzios S, Stathopoulos J, et al. Treatment of colorectal cancer with and without bevacizumab: a phase III study. Oncology 2010;78:376-81.

[10] Tebbutt NC, Wilson K, Gebski VJ, Cummins MM, Zannino D, van Hazel GA, et al. Capecitabine, bevacizumab, and mitomycin in first-line treatment of metastatic colorectal cancer: results of the Australasian Gastrointestinal Trials Group Randomized Phase III MAX Study. J Clin Oncol 2010;28:3191-8.
[11] Cunningham D, Lang I, Marcuello E, Lorusso V, Ocvirk J, Shin DB, et al. Bevacizumab plus capecitabine versus capecitabine alone in elderly patients with previously untreated metastatic colorectal cancer (AVEX): an open-label, randomised phase 3 trial. Lancet Oncol 2013;14:1077-85.

[12] Modest D, Fischer L, von Weikersthal L, Decker L, VehlingKaiser U, Uhlig J, et al. Randomized phase III study of fluoropyrimidine (FP) plus bevacizumab (BEV) vs. FP plus irinotecan (IRI) and BEV as first-line therapy for metastatic colorectal cancer (mCRC): German AIO KRK0110 (ML22011) study. Ann Oncol 2017;29(Suppl_5):v158-208.

[13] Cremolini C, Loupakis F, Antoniotti C, Lupi C, Sensi E, Lonardi $S$, et al. FOLFOXIRI plus bevacizumab versus FOLFIRI plus bevacizumab as first-line treatment of patients with metastatic colorectal cancer: updated overall survival and molecular subgroup analyses of the open-label, phase 3 TRIBE study. Lancet Oncol 2015;16:1306-15.

[14] Schmoll HJ, Meinert FM, Cygon F, Garlipp B, Junghanss C, Leithauser M, et al. "CHARTA": FOLFOX/bevacizumab vs. FOLFOXIRI/bevacizumab in advanced colorectal cancerfinal results, prognostic and potentially predictive factors from the randomized phase II trial of the AIO. J Clin Oncol 2017;35:3533.

[15] Gruenberger T, Bridgewater J, Chau I, Garcia Alfonso P, Rivoire M, Mudan S, et al. Bevacizumab plus mFOLFOX-6 or FOLFOXIRI in patients with initially unresectable liver metastases from colorectal cancer: the OLIVIA multinational randomised phase Il trial. Ann Oncol 2015;26:702-8.

[16] Falcone A, Cremolini C, Loupakis F, Lonardi S, Casagrande ME, Murgioni S, et al. FOLFOXIRI plus bevacizumab (bev) followed by maintenance with bev alone or bev plus metronomic chemotherapy (metroCT) in metastatic colorectal cancer (mCRC): the phase II randomized MOMA trial. Ann Oncol 2017;27:LBA21.

[17] Cremolini C, Marmorino F, Loupakis F, Loupakis F, Masi G, Antoniotti C, et al. TRIBE-2: a phase III, randomized, openlabel, strategy trial in unresectable metastatic colorectal cancer patients by the GONO group. BMC Cancer 2017;17:408.

[18] Bendell JC, Tan BR, Reeves JA, Xiong H, Somer BG, Lenz H-J, et al. Overall response rate (ORR) in STEAM, a randomized, open-label, phase 2 trial of sequential and concurrent FOLFOXIRI-bevacizumab (BEV) vs FOLFOX-BEV for the first-line (1L) treatment (tx) of patients (pts) with metastatic colorectal cancer (mCRC). J Clin Oncol 2016;34:492.

[19] Bokemeyer C, Kohne CH, Ciardiello F, Lenz HJ, Heinemann V, Klinkhardt $\mathrm{U}$, et al. FOLFOX4 plus cetuximab treatment and RAS mutations in colorectal cancer. Eur J Cancer 2015;51:1243-52.

[20] Van Cutsem E, Kohne CH, Hitre E, Zaluski J, Chang Chien CR, Makhson A, et al. Cetuximab and chemotherapy as initial treatment for metastatic colorectal cancer. N Engl J Med 2009;360:1408-17.

[21] Van Cutsem E, Kohne CH, Lang I, Folprecht G, Nowacki MP, Cascinu S, et al. Cetuximab plus irinotecan, fluorouracil, and leucovorin as first-line treatment for metastatic colorectal cancer: updated analysis of overall survival according to tumor KRAS and BRAF mutation status. J Clin Oncol 2011;29:2011-9.

[22] Kohne CH, Poston G, Folprecht G, Ciardiello F, Ronga P, Beier $F$, et al. FOLFIRI plus cetuximab in patients with liver-limited or non-liver-limited RAS wild-type metastatic colorectal cancer: A retrospective subgroup analysis of the CRYSTAL study. Eur J Surg Oncol 2016;42:1540-7. 
[23] Bokemeyer C, Van Cutsem E, Rougier P, Ciardiello F, Heeger S, Schlichting M, et al. Addition of cetuximab to chemotherapy as first-line treatment for KRAS wild-type metastatic colorectal cancer: pooled analysis of the CRYSTAL and OPUS randomised clinical trials. Eur J Cancer 2012;48:1466-75.

[24] Tveit KM, Guren T, Glimelius B, Pfeiffer P, Sorbye H, Pyrhonen $\mathrm{S}$, et al. Phase III trial of cetuximab with continuous or intermittent fluorouracil, leucovorin, and oxaliplatin (Nordic FLOX) versus FLOX alone in first-line treatment of metastatic colorectal cancer: the NORDIC-VII study. J Clin Oncol 2012;30:1755-62.

[25] Douillard JY, Siena S, Cassidy J, Tabernero J, Burkes R, Barugel $M$, et al. Randomized, phase III trial of panitumumab with infusional fluorouracil, leucovorin, and oxaliplatin (FOLFOX4) versus FOLFOX 4 alone as first-line treatment in patients with previously untreated metastatic colorectal cancer: the PRIME study. J Clin Oncol 2010;28:4697-705.

[26] Douillard JY, Siena S, Cassidy J, Tabernero J, Burkes R, Barugel $M$, et al. Final results from PRIME: randomized phase 3 study of panitumumab with FOLFOX4 for first-line treatment of metastatic colorectal cancer. Ann Oncol 2014;25:1346-55.

[27] Folprecht G, Gruenberger T, Bechstein WO, Raab HR, Lordick F, Hartmann JT, et al. Tumour response and secondary resectability of colorectal liver metastases following neoadjuvant chemotherapy with cetuximab: the CELIM randomised phase 2 trial. Lancet Oncol 2010;11:38-47.

[28] Folprecht G, Gruenberger T, Bechstein W, Raab HR, Weitz J, Lordick F, et al. Survival of patients with initially unresectable colorectal liver metastases treated with FOLFOX/cetuximab or FOLFIRI/cetuximab in a multidisciplinary concept (CELIM study). Ann Oncol 2014;25:1018-25.

[29] Carrato A, Abad A, Massuti B, Gravalos C, Escudero P, Longo-Munoz F, et al. First-line panitumumab plus FOLFOX4 or FOLFIRI in colorectal cancer with multiple or unresectable liver metastases: a randomised, phase II trial (PLANET-TTD). Eur J Cancer 2017;81:191-202.

[30] Primrose J, Falk S, Finch-Jones M, Valle J, O'Reilly D, Siriwardena A, et al. Systemic chemotherapy with or without cetuximab in patients with resectable colorectal liver metastasis: the new EPOC randomised controlled trial. Lancet Oncol 2014;15:601-11.

[31] Nordlinger B, Sorbye H, Glimelius B, Poston GJ, Schlag PM, Rougier P, et al. Perioperative FOLFOX4 chemotherapy and surgery versus surgery alone for resectable liver metastases from colorectal cancer (EORTC 40983): long-term results of a randomised, controlled, phase 3 trial. Lancet Oncol 2013;14:1208-15.

[32] Bridgewater J, Pugh S, Whitehead A, Stanton L, Eminton Z, Mellor J, et al. Perioperative chemotherapy with or without cetuximab in patients (pts) with resectable colorectal liver metastasis (CRLM): mature analysis of overall survival (OS) in the new EPOC randomised controlled trial. Ann Oncol 2017;8:v158-208.

[33] Ye LC, Liu TS, Ren L, Wei Y, Zhu DX, Zai SY, et al. randomized controlled trial of cetuximab plus chemotherapy for patients with KRAS wild-type unresectable colorectal liver-limited metastases. J Clin Oncol 2013;31:1931-8.

[34] Folprecht G, Hamann S, Schutte K, Trarbach T, StoehlmacherWilliams J, Ehninger G. Dose escalating study of cetuximab and 5-FU/folinic acid (FA)/oxaliplatin/irinotecan (FOLFOXIRI) in first line therapy of patients with metastatic colorectal cancer. BMC Cancer 2014;14:521.
[35] Garufi C, Torsello A, Tumolo S, Ettorre GM, Zeuli M, Campanella $C$, et al. Cetuximab plus chronomodulated irinotecan, 5-fluorouracil, leucovorin and oxaliplatin as neoadjuvant chemotherapy in colorectal liver metastases: POCHER trial. Br J Cancer 2010;103:1542-7.

[36] Assenat E, Desseigne F, Thezenas S, Viret F, Mineur L, Kramar A, et al. Cetuximab plus FOLFIRINOX (ERBIRINOX) as first-line treatment for unresectable metastatic colorectal cancer: a phase II trial. Oncologist 2011;16:1557-64.

[37] Saridaki Z, Androulakis N, Vardakis N, Vamvakas L, Kabouraki $\mathrm{E}$, Kalbakis K, et al. A triplet combination with irinotecan (CPT11), oxaliplatin (LOHP), continuous infusion 5 -fluorouracil and leucovorin (FOLFOXIRI) plus cetuximab as first-line treatment in KRAS wt, metastatic colorectal cancer: a pilot phase II trial. Br J Cancer 2012;107:1932-7.

[38] Fornaro L, Lonardi S, Masi G, Loupakis F, Bergamo F, Salvatore L, et al. FOLFOXIRI in combination with panitumumab as first-line treatment in quadruple wild-type (KRAS, NRAS, HRAS, BRAF) metastatic colorectal cancer patients: a phase II trial by the Gruppo Oncologico Nord Ovest (GONO). Ann Oncol 2013;24:2062-7.

[39] Geissler M, Martens UM, Knorrenschield R, Greeve J, Florschuetz A, Tannapfel A, et al. mFOLFOXIRI + panitumumab versus FOLFOXIRI as first-line treatment in patients with RAS wild-type metastatic colorectal cancer $m(C R C)$ : a randomized phase II VOLFI trial of the AIO (AIO-KRK0109). Ann Oncol 2017;28:4750.

[40] Heinemann V, von Weikersthal LF, Decker T, Kiani A, Vehling-Kaiser U, Al-Batran SE, et al. FOLFIRI plus cetuximab versus FOLFIRI plus bevacizumab as first-line treatment for patients with metastatic colorectal cancer (FIRE-3): a randomised, openlabel, phase 3 trial. Lancet Oncol 2014;15:1065-75.

[41] Stintzing S, Modest DP, Rossius L, Lerch MM, von Weikersthal LF, Decker T, et al. FOLFIRI plus cetuximab versus FOLFIRI plus bevacizumab for metastatic colorectal cancer (FIRE-3): a posthoc analysis of tumour dynamics in the final RAS wild-type subgroup of this randomised open-label phase 3 trial. Lancet Oncol 2016;17:1426-34.

[42] Modest DP, Denecke T, Pratschke J, Ricard I, Lang H, Bemelmans M, et al. Surgical treatment options following chemotherapy plus cetuximab or bevacizumab in metastatic colorectal cancer-central evaluation of FIRE-3. Eur J Cancer 2017;88:77-86.

[43] Venook AP, Niedzwiecki D, Lenz H-J, Innocenti F, Mahoney MR, O'Neil BH, et al. CALGB/SWOG 80405: phase III trial of irinotecan/5-FU/leucovorin (FOLFIRI) or oxaliplatin/5-FU/leucovorin (mFOLFOX6) with bevacizumab (BV) or cetuximab (CET) for patients (pts) with KRAS wild-type (wt) untreated metastatic adenocarcinoma of the colon or rectum (MCRC). J Clin Oncol 2014;32:LBA3.

[44] Lenz H, Niedzwiecki D, Innocenti F, Blanke C, Mahony MR, O'Neil BH, et al. CALGB/SWOG 80405: phase III trial of irinotecan/5-FU/leucovorin (FOLFIRI) or oxaliplatin/5-FU/ leucovorin (mFOLFOX6) with bevacizumab (BV) or cetuximab (CET) for patients (pts) with expanded RAS analyses untreated metastatic adenocarcinoma of the colon or rectum (MCRC). Ann Oncol 2014;25:mdu438.13.

[45] Schwartzberg LS, Rivera F, Karthaus M, Fasola G, Canon JL, Hecht JR, et al. PEAK: a randomized, multicenter phase II study of panitumumab plus modified fluorouracil, leucovorin, and oxaliplatin 
(mFOLFOX6) or bevacizumab plus mFOLFOX6 in patients with previously untreated, unresectable, wild-type KRAS exon 2 metastatic colorectal cancer. J Clin Oncol 2014;32:2240-7.

[46] Yamauchi M, Morikawa T, Kuchiba A, Imamura Y, Qian ZR, Nishihara R, et al. Assessment of colorectal cancer molecular features along bowel subsites challenges the conception of distinct dichotomy of proximal versus distal colorectum. Gut 2012;61:847-54.

[47] Loupakis F, Yang DY, Yau L, Feng SB, Cremolini C, Zhang W, et al. Primary tumor location as a prognostic factor in metastatic colorectal cancer. J Natl Cancer Inst 2015;107:pii:dju427.

[48] Tejpar S, Stintzing S, Ciardiello F, Tabernero J, Van Cutsem E, Beier $\mathrm{F}$, et al. Prognostic and predictive relevance of primary tumor location in patients with RAS wild-type metastatic colorectal cancer: retrospective analyses of the CRYSTAL and FIRE-3 trials. JAMA Oncol 2016. doi: 10.1001/jamaoncol.2016.3797. [Epub ahead of print].

[49] Arnold D, Lueza B, Douillard JY, Peeters M, Lenz HJ, Venook A, et al. Prognostic and predictive value of primary tumour side in patients with RAS wild-type metastatic colorectal cancer treated with chemotherapy and EGFR directed antibodies in six randomized trials. Ann Oncol 2017;28:1713-29.

[50] Petrelli F, Tomasello G, Borgonovo K, Ghidini M, Turati L, Dallera $\mathrm{P}$, et al. Prognostic survival associated with left-sided vs. right-sided colon cancer: a systematic review and metaanalysis. JAMA Oncol 2016. doi: 10.1001/jamaoncol.2016.4227. [Epub ahead of print].

[51] Van Cutsem E, Cervantes A, Adam R, Sobrero A, Van Krieken JH, Aderka D, et al. ESMO consensus guidelines for the management of patients with metastatic colorectal cancer. Ann Oncol 2016;27:1386-422.

[52] Di Nicolantonio F, Martini M, Molinari F, Sartore-Bianchi A, Arena S, Saletti P, et al. Wild-type BRAF is required for response to panitumumab or cetuximab in metastatic colorectal cancer. J Clin Oncol 2008;26:5705-12.

[53] Saridaki Z, Tzardi M, Papadaki C, Sfakianaki M, Pega F, Kalikaki A, et al. Impact of KRAS, BRAF, PIK3CA mutations, PTEN, AREG, EREG expression and skin rash in $\geq 2$ line cetuximab-based therapy of colorectal cancer patients. PLoS One 2011;6:e15980.
[54] Souglakos J, Philips J, Wang R, Marwah S, Silver M, Tzardi M, et al. Prognostic and predictive value of common mutations for treatment response and survival in patients with metastatic colorectal cancer. Br J Cancer 2009;101:465-72.

[55] Perrone F, Lampis A, Orsenigo M, Di Bartolomeo M, Gevorgyan A, Losa M, et al. PI3KCA/PTEN deregulation contributes to impaired responses to cetuximab in metastatic colorectal cancer patients. Ann Oncol 2009;20:84-90.

[56] Sartore-Bianchi A, Martini M, Molinari F, Veronese S, Nichelatti $\mathrm{M}$, Artale $\mathrm{S}$, et al. PIK3CA mutations in colorectal cancer are associated with clinical resistance to EGFR-targeted monoclonal antibodies. Cancer Res 2009;69:1851-7.

[57] Prenen H, De Schutter J, Jacobs B, De Roock W, Biesmans B, Claes B, et al. PIK3CA mutations are not a major determinant of resistance to the epidermal growth factor receptor inhibitor cetuximab in metastatic colorectal cancer. Clin Cancer Res 2009;15:3184-8.

[58] Pietrantonio F, Petrelli F, Coinu A, Di Bartolomeo M, Borgonovo K, Maggi C, et al. Predictive role of BRAF mutations in patients with advanced colorectal cancer receiving cetuximab and panitumumab: a meta-analysis. Eur J Cancer 2015;51:587-94.

[59] Rowland A, Dias MM, Wiese MD, Kichenadasse G, McKinnon RA, Karapetis CS, et al. Meta-analysis of BRAF mutation as a predictive biomarker of benefit from anti-EGFR monoclonal antibody therapy for RAS wild-type metastatic colorectal cancer. Br J Cancer 2015;112:1888-94.

[60] Leitlinienprogramm Onkologie (Deutsche Krebsgesellschaft DK, AWMF). S3-Leitlinie Kolorektales Karzinom, Langversion 2.0. In; 2017.

[61] Benson AB, 3rd, Venook AP, Cederquist L, Chan E, Chen Y], Cooper HS, et al. Colon Cancer, Version 1.2017, NCCN Clinical Practice Guidelines in Oncology. J Natl Compr Canc Netw 2017;15:370-98.

Supplemental Material: The article (https://doi.org/10.1515/iss2018-0012) offers reviewer assessments as supplementary material. 


\section{Reviewer Assessment}

Arndt Vogel* and Martha M. Kirstein

\section{First-line molecular therapies in the treatment of metastatic colorectal cancer - a literature-based review of phases II and III trials}

https://doi.org/10.1515/iss-2018-0012

Received March 23, 2018; accepted April 3, 2018

*Corresponding author: Prof. Dr. med. Arndt Vogel, Department of Gastroenterology, Hepatology and Endocrinology, Hannover Medical School, Carl-Neuberg-Str. 1, 30625 Hannover, Germany, Phone: 00495325119590, E-mail: vogel.arndt@mh-hannover.de

\section{Reviewers' Comments to Original Submission}

\section{Reviewer 1: anonymous}

Mar 26, 2018

Reviewer Recommendation Term:

Overall Reviewer Manuscript Rating:

Custom Review Questions

Is the subject area appropriate for you?

Does the title clearly reflect the paper's content?

Does the abstract clearly reflect the paper's content?

Do the keywords clearly reflect the paper's content?

Does the introduction present the problem clearly?

Are the results/conclusions justified?

How comprehensive and up-to-date is the subject matter presented?

How adequate is the data presentation?

Are units and terminology used correctly?

Is the number of cases adequate?

Are the experimental methods/clinical studies adequate?

Is the length appropriate in relation to the content?

Does the reader get new insights from the article?

Please rate the practical significance.

Please rate the accuracy of methods.

Please rate the statistical evaluation and quality control.

Please rate the appropriateness of the figures and tables.

Please rate the appropriateness of the references.

Please evaluate the writing style and use of language.

Please judge the overall scientific quality of the manuscript.

Are you willing to review the revision of this manuscript?

Accept
80
Response
3
3
4
4
4
4
5 - High/Yes
4
N/A
N/A
N/A
4
4
4
N/A
N/A
N/A
4
4
4
Yes

Accept 


\section{Comments to Authors:}

This is a nice overview of the possibilities of monoclonal therapies in advanced colorectal cancer. One or two tables with the relevant data coud have improved the manuscript in order to improve the visibility of the data. For the title I suggest: ...A literature based Review... instead of ...study.

\section{Reviewer 2: anonymous}

Mar 31, 2018

\section{Reviewer Recommendation Term:}

Overall Reviewer Manuscript Rating:

\section{Custom Review Questions}

Is the subject area appropriate for you?

Does the title clearly reflect the paper's content?

Does the abstract clearly reflect the paper's content?

Do the keywords clearly reflect the paper's content?

Does the introduction present the problem clearly?

Are the results/conclusions justified?

How comprehensive and up-to-date is the subject matter presented?

How adequate is the data presentation?

Are units and terminology used correctly?

Is the number of cases adequate?

Are the experimental methods/clinical studies adequate?

Is the length appropriate in relation to the content?

Does the reader get new insights from the article?

Please rate the practical significance.

Please rate the accuracy of methods.

Please rate the statistical evaluation and quality control.

Please rate the appropriateness of the figures and tables.

Please rate the appropriateness of the references.

Please evaluate the writing style and use of language.

Please judge the overall scientific quality of the manuscript.

Are you willing to review the revision of this manuscript?

\section{Accept}

80

\section{Response}

4

4

4

4

4

4

4

3

$\mathrm{N} / \mathrm{A}$

$\mathrm{N} / \mathrm{A}$

4

4

4

3

4

$\mathrm{N} / \mathrm{A}$

4

4

4

Yes

Comments to Authors:

No comments 